\title{
The perception of lightness in 3-D curved objects
}

\author{
LUIZ PESSOA \\ Federal University of Rio de Janeiro, Rio de Janeiro, Brazil \\ ENNIO MINGOLLA \\ Boston University, Boston, Massachusetts \\ and \\ LAWRENCE E. AREND \\ Universität Bielefeld, Bielefeld, Germany
}

\begin{abstract}
Lightness constancy in complex scenes requires that the visual system take account of information concerning variations of illumination falling on visible surfaces. Three experiments on the perception of lightness for three-dimensional (3-D) curved objects show that human observers are better able to perform this accounting for certain scenes than for others. The experiments investigate the effect of object curvature, illumination direction, and object shape on lightness perception. Lightness constancy was quite good when a rich local gray-level context was provided. Deviations occurred when both illumination and reflectance changed along the surface of the objects. Does the perception of a 3-D surface and illuminant layout help calibrate lightness judgments? Our results showed a small but consistent improvement between lightness matches on ellipsoid shapes, relative to flat rectangle shapes, under illumination conditions that produce similar image gradients. Illumination change over 3-D forms is therefore taken into account in lightness perception.
\end{abstract}

Lightness is the component of perceived surface color that varies from black through gray to white. Historically, lightness constancy has been treated as the approximate invariance of achromatic surface color with changes in the overall illuminance of a scene. However, changes in illumination represent only one of several obstacles to perceiving a given surface material as having constant achromatic surface color. Schirillo and Arend (1995) describe three environmental challenges to constancy: (1) change in illumination intensity (also known as Type I), (2) change in background (Type II), and (3) change in shape (which we could call Type III). The present paper discusses the perception of lightness in three-dimensional (3-D) surfaces that "curve in depth."

Several studies have provided evidence that human observers display a high degree of lightness constancy under changes in illumination (Arend \& Goldstein, 1987, 1990; Jacobsen \& Gilchrist, 1988). Fewer studies exist for constancy under changes of background, though it is as important to the visual system as constancy across different illuminations. As Whittle (1994, p. 128) puts it, if some

L.P. was supported in part by the Air Force Office of Scientific Research (AFOSR F49620-92-J-0334) and the Office of Naval Research (ONR N00014-J-4100); E.M. was supported in part by the Office of Naval Research (ONR N00014-94-1-0597). The authors thank the subjects for helping collect the results shown here, and Alan Gilchrist, Jacob Beck, and an anonymous reviewer for many helpful comments on earlier versions of the manuscript. Correspondence should be addressed to L. Pessoa, Programa de Engenharia de Sistemas e ComputacaoCOPPE Sistemas, Universidade Federal do Rio de Janeiro, Centro de Tecnologia, Bloco H, Sala H-319, Ilha do Fundao, Rio de Janeiro, RJ 21945-970, Brazil (e-mail: pessoa@cos.ufrj.br). form of background constancy did not exist, "objects would flash on and off all the time as they changed from being increments to being decrements. They do not." Recent experiments by Whittle (1992) and Arend and Spehar (1993b) show that the effects of the background are small (see also Arend, 1994). For example, changing the background from black to white causes a fixed, small deviation from constancy (less than 1.5 Munsell value steps; see Gilchrist, 1988; Gilchrist, Delman, \& Jacobsen, 1983).

Recent studies have shown that the perception of 3-D curved shapes interacts with lightness perception. Knill and Kersten (1991) showed that the lightness of a stimulus may be affected by the shape of its outline contour (see also Buckley, Frisby, \& Freeman, 1994; Pessoa, Grunewald, \& Ross, 1995; Ramachandran, 1988). The present series of experiments probes the extent to which the visual system attains lightness constancy given changes in shape. More precisely, constancy is investigated by having test and standard patches vary in placement on a curved surface. To our knowledge, this is the first study to assess the extent of lightness constancy across locations on the smoothly curved surface of an object that appears 3-D (see Figure 1).

For 3-D scenes, overall illumination may vary in different parts of the scene due to (1) local orientation of surface with respect to light source and (2) distance from light source to surface. Variations in 3-D orientation and depth of surfaces of constant albedo may produce extreme variations in the luminance of different regions. For example, in Figure 2, Regions A and B are made of the same material but have different illuminations. In fact, Re- 


\begin{tabular}{|l|l|}
\hline $\begin{array}{l}\text { Judge lightness with } \\
\text { changes in }\end{array}$ & Performance \\
\hline Overall illumination level & Excellent \\
Background & Good \\
Shape & Present study \\
\hline
\end{tabular}

Figure 1. Three environmental challenges to perceiving a given surface as having constant achromatic surface color.

gion B may appear very dim since the surface normal is nearly perpendicular to the light source (assuming a Lambertian lighting model of surface reflectance and no ambient illumination).

In scenes with spatial-illumination gradients, the visual system must be able to discriminate between reflectance and illumination gradients. Moreover, in order to produce constancy, the system must accurately parse, or decompose, local luminance gradients into illumination and reflectance components. Arend and Goldstein's (1990) experiments with unevenly illuminated Mondrian displays revealed excellent lightness constancy - even though the luminance gradients were clearly visible-showing that the visual system is able, under some circumstances, to appropriately perform such operations. To some extent, perceiving curved objects as having constant lightness is similar to being able to perceive constant lightness in flat displays with uneven illumination. In both, luminance gradients may be present for surfaces of constant albedo. However, for 3-D scenes, the variation of the illumination is often in multiple directions, as, for example, for a sphere. For flat surfaces, shading variation in the second dimension is negligible except in the relatively rare case of a small light source close to the surface.

Another way in which 3-D scenes differ from 2-D scenes with uneven illumination is that information concerning 3-D orientation and depth is available. One of the questions addressed in this study was whether the perception of surface and illuminant layout help calibrate lightness judgments. In other words, is the illumination change over 3-D forms taken into account in lightness perception? In the present article, this was investigated by comparing lightness matches for objects in which illumination was consistent with surface geometry (ellipsoids) and objects in which this did not occur (rectangles). For both ellipsoids and rectangles, illumination conditions were similar, but only for the former did the illumination conform to the geometry (bounding contour) of the object.

In Arend and Goldstein's (1990) unevenly illuminated displays, both standard and test patches were surrounded by regions having the same reflectance. Thus, lightness constancy may have resulted from employing a strategy of equating local luminance ratios. In the present experiments, we studied the perception of lightness in 3-D curved objects that produce highly unevenly illuminated scenes, while, at the same time, controlling for the potential strategy of equating local ratios.

\section{GENERAL METHOD}

\section{Equipment}

Stimuli were generated and presented on a carefully calibrated Silicon Graphics workstation. The subjects adjusted the appearance of the test patch by using a dial (see below). Between trials, the computer randomly offset the relationship between dial position and test appearance to prevent position cues from influencing the adjustments.

\section{Stimuli}

All stimuli were simulations of simple scenes. Two types of objects were used: ellipsoids and flat rectangles. Both shape and orientation of ellipsoids were governed by appropriate choices of coefficients in the following equation for general quadric surfaces:

$$
\begin{aligned}
a_{11} x^{2} & +a_{22} y^{2}+a_{33} z^{2}+2 a_{12} x y+2 a_{13} x z \\
& +2 a_{23} y z+2 a_{14} x+2 a_{24} y+2 a_{34} z+a_{44}=0 .
\end{aligned}
$$

Equation 1 was also used to determine the surface normal at surface points used by the shading algorithm of the graphical routines available on the workstation. The lighting model employed was Lambertian, with a small ( $5 \%$ ) component of ambient illumination. The ambient illumination prevented image regions from having luminance levels too close to zero.

In order to maintain the display conditions for ellipsoids and rectangles as similar as possible, the surface normals along the horizontal midline of a given ellipsoid were used to define the shading of the corresponding rectangle. Therefore, the 1-D luminance cross-section along the midline was identical for both shapes. For rectangles, the normals were replicated vertically, producing an illumination consistent with either a spatially varying illumination on a planar surface or a cylindrical shape whose cross-sections corresponded to that of the horizontal midline of the ellipsoid. Figure 3 shows some examples of stimuli used in the present study.

Ellipsoids were generated by using Equation 1 with the following coefficients: $a_{11}=1.0, a_{22}=1.78$, and $a_{33}=1.56$ or 0.32 , depending on the level of curvature (see below). All remaining coefficients were zero.

\section{Procedure}

The subjects were told that the displays simulated unevenly illuminated objects and that the illumination originated from the right.

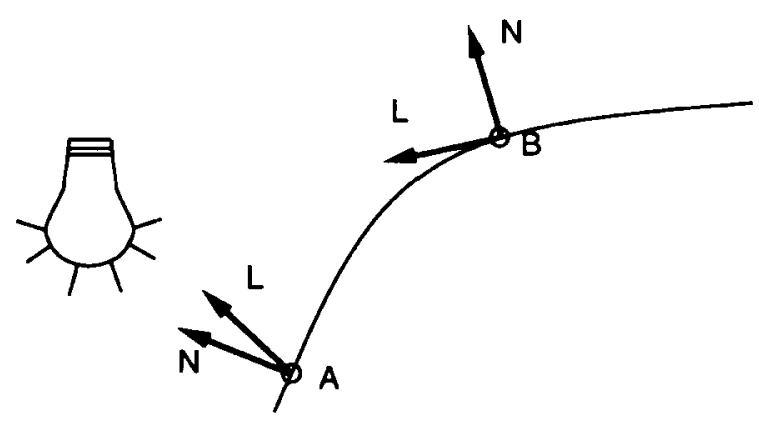

Figure 2. For 3-D scenes, overall illumination may vary in different parts of the scene. Regions $A$ and $B$ have the same reflectance but different illuminations. $N$ is the unit vector normal to the surface, and $L$ is the vector in the direction of the light source. For a Lambertian model, the intensity at a point is proportional to the cosine of the angle between vectors $N$ and $L$. 

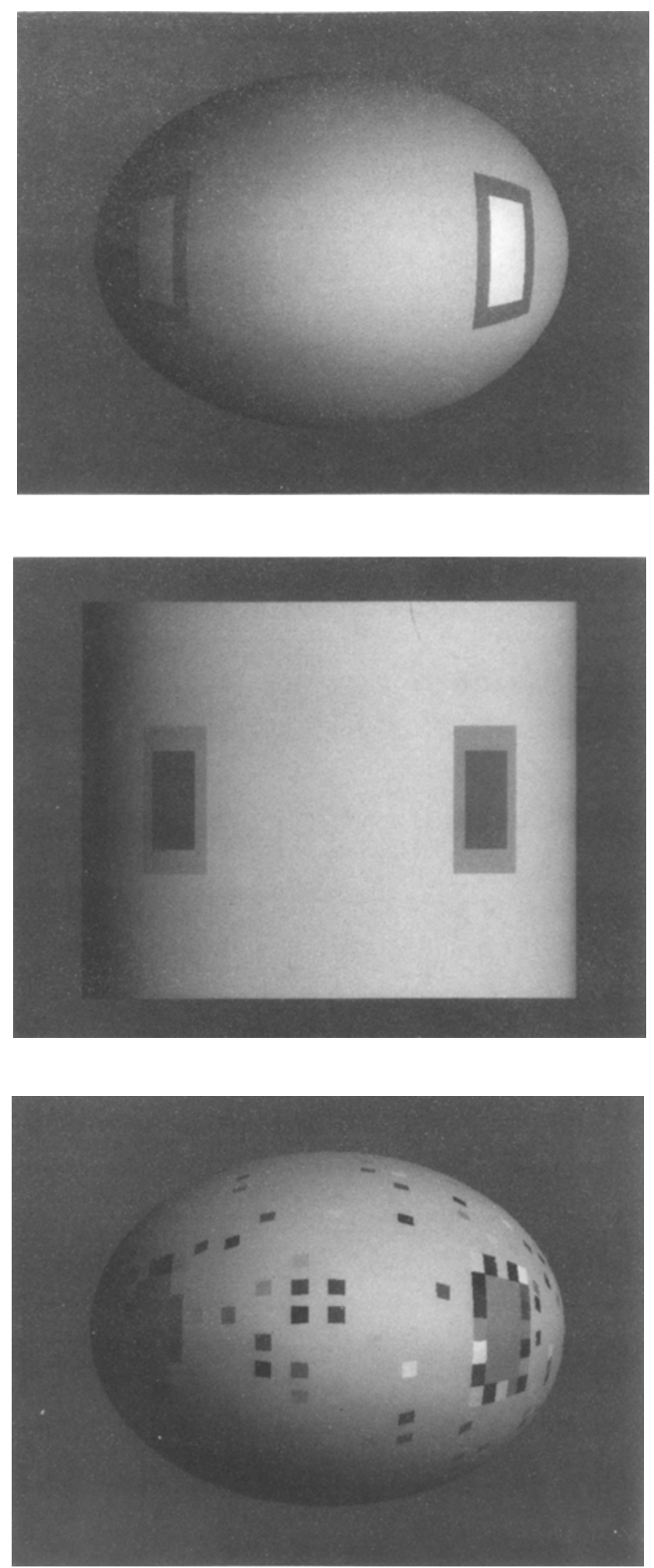

Figure 3. Examples of stimuli used in the experiments. The test (adjustable) patch is located on the right of the objects; the standard (fixed) patch is located on the left. Top: Ellipsoid used in Experiment 1 (high curvature, high reflectance standard, side illumination). Middle: Rectangle used in Experiment 1 (low curvature, low reflectance standard, side illumination). Bottom: Ellipsoid used in Experiment 2 (high curvature, medium reflectance standard, elevated illumination). It should be noted that because of distortions in the process of photographic reproduction, these pictures appear considerably less realistic and three-dimensional than they do on a video screen. 
They were instructed to make the test patch "look as if cut from the same piece of paper" as the standard patch (i.e., to perform lightness matches). In the conditions involving uniform-reflectance standard and test surrounds, they were told that these were not of the same reflectance (see below). It was then explained to them that, after adjustment, the apparent contrast of the test and standard relative to their surrounds needed not appear the same.

The display was viewed binocularly ${ }^{1}$ at a distance of $50 \mathrm{~cm}$. Lightness matches were performed in a dark room. Displays were continuously presented, and the subjects matched the test patch to the standard patch by using a dial to vary test-patch appearance; no fixation was required. Internally, the dial controlled the reflectance of the test patch within the lighting model. Thus, when rendered, the test patch also exhibited luminance gradients (see Figure 4).

\section{Subjects}

Three subjects (25-30 years of age), with normal or correctedto-normal vision, served as observers. Subject A.G. participated in all three experiments, Subject L.P. participated in Experiments 1 and 2, and Subject N.M. participated in Experiments 2 and 3. Subject L.P. was one of the authors; Subjects N.M. and A.G. were naive with respect to the purpose of the experiment. Subject L.P was experienced in lightness matching; Subjects A.G. and N.M. had no prior experience in lightness matching but were familiar with psychophysical testing.

\section{EXPERIMENT 1 Center-Surround Patches}

Lightness perception on 3-D ellipsoids was studied by varying object curvature and illumination direction. Wallach's (1948) ratio hypothesis states that equal local luminance ratios produce equal lightnesses. Wallach's hypothesis holds in the very special case of patches on equal-reflectance surrounds but fails under more general conditions (e.g., Arend \& Spehar, 1993a, 1993b). One question addressed in the present experiment was whether the perception of surface and illuminant layout helps calibrate lightness judgment and prevent misjudgment based on local contrast ratios of luminance. Moreover, we wanted to assess whether the illumination change over 3-D forms is taken into account in lightness perception.

\section{Method}

There were four experimental factors: shape, curvature, illumination direction, and reflectance of standard patch.

The object shapes in the graphic model were either ellipsoids or flat rectangles with similar illumination conditions, such that the 1-D luminance cross-section along the midline was identical for both shapes (see General Method section). As stated, the shading on the rectangles was equivalent to the shading of a cylinder of crosssection identical to the ellipsoid or to that produced by a spatially varying illumination on a planar surface. At the same time, the geometry (bounding contour) of the object was rectangular, not cylindrical. From generic viewpoints, the bounding contour of the 3-D cylinder would be curved, and one end surface of the cylinder should be visible. The rectangular contour of our pattern is consistent with a section of a cylinder only if the eye is at a special viewpoint (i.e., exactly on the line of intersection of the tilted end planes of the object). The visual system often avoids such "nongeneric" interpretations in favor of more generic ones (Nakayama \& Shimojo, 1990).

Two levels of curvature were used by manipulating Equation 1 . More curved objects implied a steeper gradient and a larger difference in illumination for the test and standard regions.

Illumination was either from the side or elevated. Side illumination was obtained by having the light source displaced horizontally to the right of the object. The position of the light source can be expressed in polar coordinates by the angles $\theta=26.56^{\circ}$ (rotation in the $x y$ plane) and $\phi=0.0^{\circ}$ (rotation in the $y z$ plane). ${ }^{2}$ In the elevated condition, both horizontal and vertical displacements were used. For lowcurvature objects, $\theta=37.99^{\circ}$, and $\phi=39.80^{\circ}$; for high-curvature objects, $\theta=30.25^{\circ}$, and $\phi=30.98^{\circ}$.

Lightness matches were performed for standard patches assuming one of three reflectance values: low $(R=0.30)$, medium $(R=0.50)$, and high $(R=0.95) .{ }^{3}$ Note that there was always an ample range of available settings above and below the physical reflectances; the lighting model actually accepted reflectances above $R=1.0 .^{4}$

Ellipsoids and rectangles had uniform albedo $(R=0.75)$. The test and standard regions were surrounded by a small region of constant albedo, forming a center-surround configuration (see Figure 3 ). To avoid local luminance ratio equality, these surround values had different reflectances and varied with the three reflectances of the standard patch. For the low standard, the test surround was $R=0.5$, and the standard surround was $R=0.6$. For the medium standard, the test surround was $R=0.1$, and the standard surround was $R=0.2$. For the high standard, the test surround was $R=0.4$, and the standard surround was $R=0.5$.
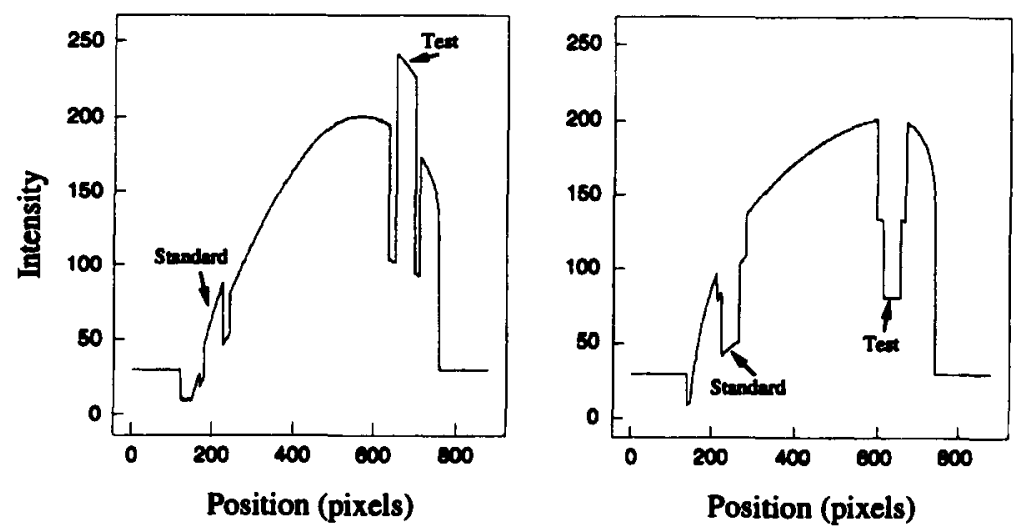

Figure 4. Examples of luminance profiles used in Experiment 1 (in arbitrary units). Both profiles are for side-illuminated objects. Left: High curvature and high reflectance standard. Right: Low curvature and low reflectance standard. In both cases, the reflectances of the test patches were set to the same value as the standard patches. 
Figure 4 shows the 1-D cross-section through the horizontal midline of two objects used in Experiment 1. The high curvature of the stimulus shown on the left produces a steep luminance gradient and a large difference in the luminance of the test and standard patches. The less extreme curvature of the stimulus on the right produces a shallower gradient and more similar luminances for the test and standard patches.

For Experiments 1-3, the horizontal and vertical major axes of the ellipsoids subtended $5.8^{\circ}$ and $4.1^{\circ}$ for a low-curvature object and $6.2^{\circ}$ and $4.3^{\circ}$ for a high-curvature object. Rectangles subtented $6.4^{\circ}$ horizontally and $4.8^{\circ}$ vertically. For ellipsoids, test and standard regions were rectangular patches projected on the surfaces (see Figure 3). On low-curvature ellipsoids, they subtended roughly $0.40^{\circ}$ $\times 1.03^{\circ}$. On high-curvature ellipsoids, they subtended roughly $0.43^{\circ}$ $\times 1.30^{\circ}$. For rectangle objects, test and standard patches subtended $0.50^{\circ} \times 1.17^{\circ}$. In all cases, test and standard patches were symmetrically placed relative to the midline of the objects.

\section{Results and Discussion}

Lightness matches for Experiment 1 are shown in Figure 5. The dotted lines represent perfect constancy lines and represent the reflectances of the standard patches. Overall, small systematic deviations from constancy occurred. For example, Subject A.G. matched the $R=0.95$ standard to an $R=0.70$ test (mean setting) for a rectangle of high curvature and elevated illumination, corresponding to an error of the order of one Munsell value step. For the low-reflectance standard, deviations also occurred. Subject L.P. tended to underestimate the standard reflectance, whereas Subject A.G. went from small overestimations for low-curvature ellipsoids to small underestimations for rectangles with elevated illumination and high curvature. For the medium-reflectance standard, lightness matches followed reflectance more closely.

The Munsell values provided in all data plots were included as a reference in order to help quantify potential deviations from lightness constancy (and these are the values one would use if one were to construct the stimuli from papers and lights). As discussed below, they should be used mainly to assess the magnitude of the deviations and not as an indication of the absolute lightness of the patches being matched.

Differences between matches for ellipsoids and rectangles were small. However, the effect of shape was statistically significant ${ }^{5}$ for Subject L.P. $[F(1,72)=20.43, p<$ $.0001]$ and for Subject A.G. $[F(1,48)=11.65, p<.0015]$. For example, for Subject A.G., ellipsoids produced smaller underestimations of the $R=0.95$ standard (compare the first pair of points with the third, and the second with the fourth). The effect of illumination direction was not statistically significant for the 2 subjects. Curvature was significant for Subject L.P. $[F(1,72)=4.41, p<.05]$ and for Subject A.G. $[F(1,48)=28.20, p<.0001]$.

It may be argued that the subjects were somehow unable to estimate reflectance in our displays, and they resorted to the strategy of determining lightness according to local luminance ratios between standard and surround and test and surround. If the subjects were using local ratios, the predicted settings would have been $R=0.25$ for the $R=0.30$ standard, $R=0.25$ for the $R=0.50$ standard, and $R=0.76$ for the $R=0.95$ standard. While such set-

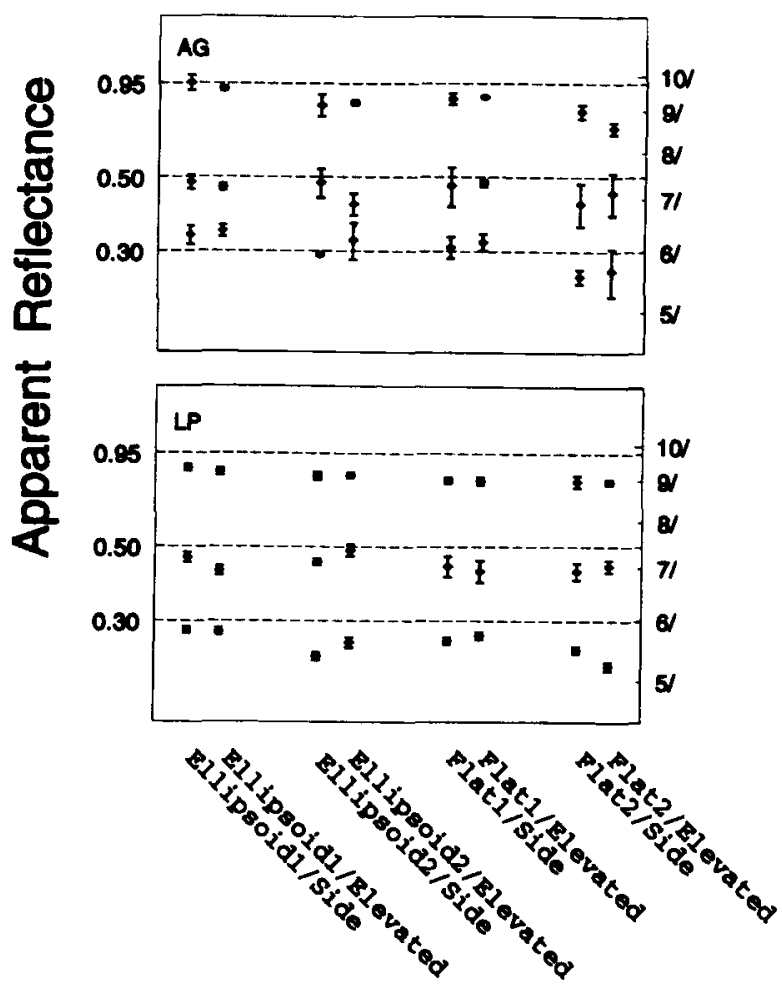

Figure 5. Experiment 1. Lightness matches in log coordinates as a function of stimulus condition for Subjects L.P. and A.G. Lightness settings are given in terms of the reflectance of the test patch. Munsell values are given on the right for reference. Ellipsoid and Flat indicate stimulus shape-ellipsoid and rectangle, respectively. The numbers 1 and 2 represent low and high curvature, respectively. Side and Elevated indicate the illumination direction. Matches are the mean of several presentations; $n=4$ for Subject L.P., and $n=3$ for Subject A.G. Error bars denote \pm 1 standard error.

tings may better account for the performance in some conditions, such as some with the high-reflectance standard, they would clearly predict large deviations for the $R=$ 0.5 standard. As Figure 5 shows, for this condition, the subjects obtained very good matches, far from the ratio prediction of $R=0.25$. Thus, we can conclude that our subjects were not using local ratios to predict lightness. Moreover, as the present discussion illustrates, local ratios do not lead to lightness constancy settings. In short, the strict version of Wallach's ratio hypothesis is incorrect (see also Arend \& Spehar, 1993a, 1993b).

In the present series of experiments no brightness matches were collected. Several investigations have shown that brightness matches are not illumination invariant (e.g., Arend \& Goldstein, 1987; Jacobsen \& Gilchrist, 1988; Schirillo, Reeves, \& Arend, 1990). Moreover, for increment conditions where the test region is immediately surrounded by a lower reflectance region, brightness matches follow luminance rather closely. This situation should be contrasted to the measurements of Experiment 1 . For example, for the high-curvature ellipsoid with side illumination, Subject L.P. matched the $R=0.95$ standard to an $R=0.81$ test (mean match). While the 
standard had a luminance of $2.95 \mathrm{fL}$, the test was set to $11.83 \mathrm{fL}$. Conversely, to equate luminance, the subject would have to have chosen a test of around $R=0.25$. $^{6}$ Such a match is clearly an unlikely lightness match since the test region would be darker than its immediate surround, unlike the standard region that is an increment. A systematic comparison between the results of Experiment 1 and the results predicted by luminance matches is shown in Figure 6. For all conditions, actual lightness matches are presented (as in Figure 5), together with the predicted reflectances given by luminance matches (see Note 6). For example, for high-curvature objects with low-reflectance test patches, luminance matches would have produced settings of $R=0.09$.

Figure 6 also provides an effective way to assess the degree of constancy for Experiment 1. First, luminance settings would produce much lower lightness matches. Second, large variations in the luminance of a test patch produce small differences in lightness settings. For example, compare the mean settings of Subjects A.G. and L.P. for the $R=0.95$ standard patch on ellipsoids to the corresponding luminance matches (first and second pair of triangles). The small decreases in apparent reflectance

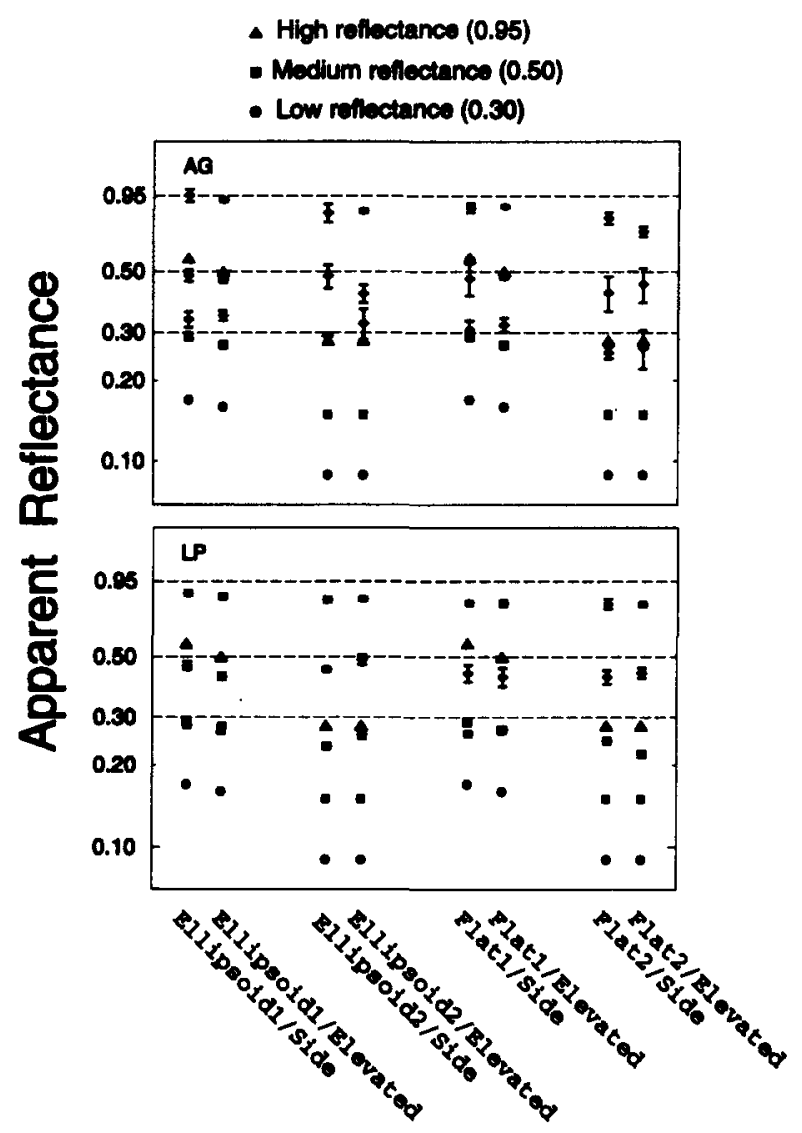

Figure 6. Experiment 1. Lightness matches in log coordinates as a function of stimulus condition for Subjects A.G. and L.P. (as in Figure 5). Values predicted by luminance matches are indicated by the solid symbols. Note the different plotting range used in the present figure. Error bars denote \pm 1 standard error. (see Subject A.G.) in no way compare to the large decreases that would be produced by luminance matches (from a match of roughly $R=0.50$ to a match of less than $R=0.30$ ). The dramatic discrepancy between the subjects' data and luminance matches shows that the subjects' settings were much closer to estimating reflectance than luminance. In conclusion, despite the steep, and visible, luminance gradients in our patterns, the subjects exhibited good lightness constancy. (The luminance predictions in Figure 6 are the same for all experiments and thus can be used for Experiments 2 and 3.)

\section{EXPERIMENT 2 Mondrian Surrounds}

The displays used in Experiment 1 were composed of only five materials, or reflectances: test, standard, test surround, standard surround, and background. The possibility exists that the displays were too impoverished to allow for best performance. In Experiment 2, test and standard regions were surrounded by small gray patches, spanning the range from black to white.

\section{Method}

The same four experimental factors and levels used in Experiment 1 were repeated in Experiment 2 (shape, curvature, illumination direction, and reflectance of standard patch). For Subjects A.G. and L.P., a low standard of $R=0.25$ was used.

In order to create a rich gray-level context for both the test and the standard regions, the uniform gray used for the immediate surrounds in Experiment 1 was replaced by a set of 20 small (roughly $0.15^{\circ} \times 0.15^{\circ}$ ) gray patches of reflectances ranging from 0.0 to $1.0 .^{7}$ These "Mondrian" surrounds were randomly selected for the test and standard surrounds. Figure 3 shows an ellipsoid employed in Experiment 2.

Both ellipsoids and rectangles were also sparsely textured with a collection of small gray patches of the same size used for the surrounds. Texture patches had reflectances randomly assigned from the range $0.0-1.0$. On ellipsoids, these were drawn on the surface of the solids, providing a stronger 3-D percept. At the same time, the texture patches provided a richer gray-level context throughout the displays.

\section{Results and Discussion}

The results of Experiment 2 are shown in Figure 7. For all subjects, lightness matches followed the constant reflectance lines very well. Small deviations can be seen, but, overall, very good lightness constancy was attained. For example, for the high-reflectance standard, the largest deviation for Subject A.G. was a (mean) match of $R=0.89$ (an error less than half a Munsell step). For the low-reflectance standard, the largest deviation for Subject L.P. was a (mean) match of $R=0.18$ (an error on the order of 1.25 Munsell steps). The effect of object shape was not significant for Subject A.G. $[F(1,48)=1.59, p>$ .20] (his performance was good throughout) but was significant for Subjects N.M. $[F(1,72)=5.92, p<.02]$ and L.P. $[F(1,48)=8.06, p<.007]$.

The use of Mondrian surrounds in Experiment 2 provided a rich local gray-level context that allowed very good lightness constancy. When the test and standard patches 


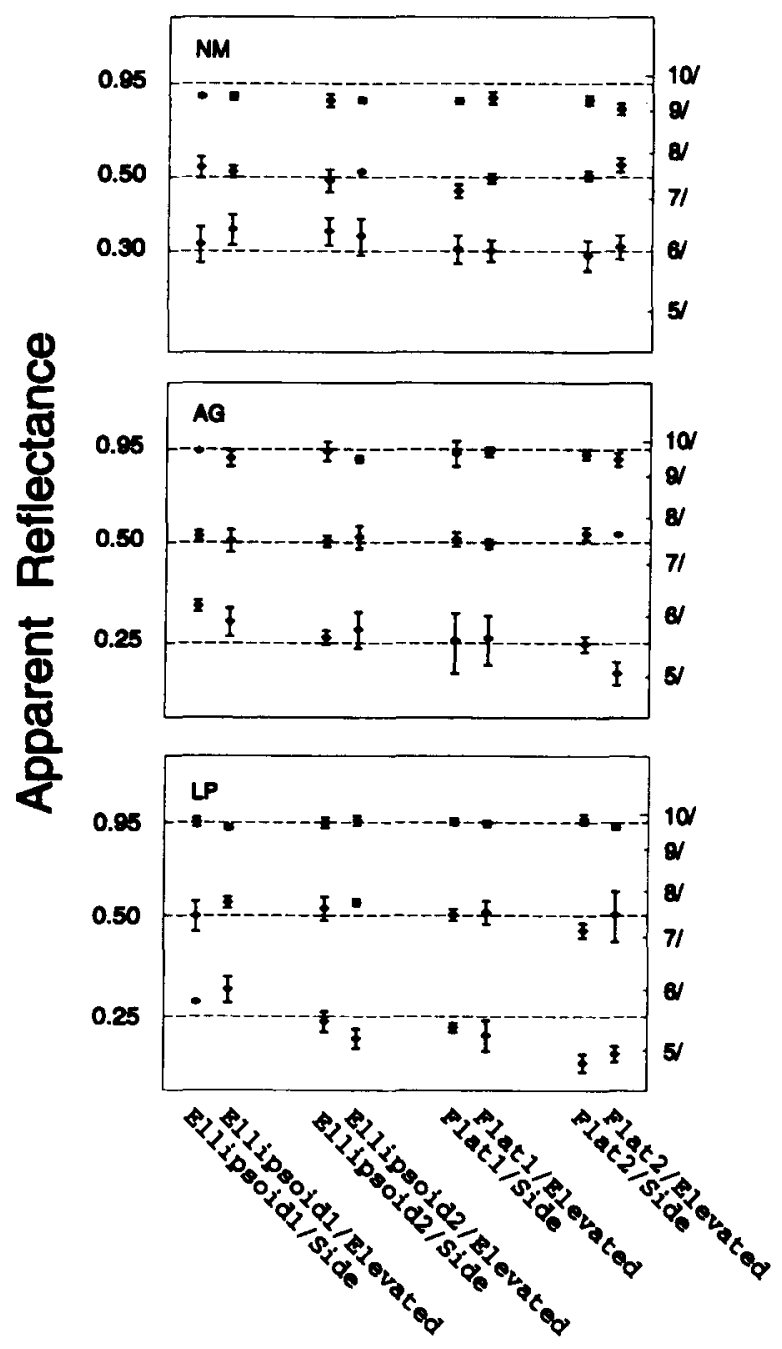

Figure 7. Experiment 2. Lightness matches in log coordinates as a function of stimulus condition for Subjects N.M., A.G., and L.P. Munsell values are given on the right for reference. Conditions as in Figure 5. Matches are the mean of several presentations; $n=4$ for Subject N.M., and $n=3$ for Subjects A.G. and L.P. Error bars denote \pm 1 standard error.

were embedded in Mondrians, their apparent gray values (lightnesses) were evaluated in relation to the gray scale defined by the Mondrian. It is possible that the texture patches throughout the objects also contributed to the good performance by allowing a better assessment of object shape and overall illumination change.

Katz (1935) argued that the greater the articulation within an illumination frame of reference, the higher the degree of lightness constancy. Articulation can be defined as the number of different gray levels within a frame of reference. Recently, Gilchrist and Cataliotti (1995; see also Gilchrist et al., 1995) have shown that articulation improves lightness constancy even when the luminance range is kept constant across stimuli with varying number of surfaces. Thus, it is also possible that a higher degree of articulation is involved in the better constancy of Experiment 2. In all, further experiments with our objects are needed to determine the roles of the (1) number of different gray levels (articulation), (2) range of gray levels, and (3) shape and illumination perception.

\section{EXPERIMENT 3 Intervening Stripes}

Parsing luminance gradients into illumination and reflectance components is central to the capability of achieving lightness constancy in unevenly illuminated scenes. In Experiment 3, we probed how well the visual system is able to accomplish such a task in our displays by interposing several uniform reflectance stripes between the test and standard regions; a single stripe ran vertically from bottom to top of the objects. Figure 8 shows a crosssection of one of the stimuli used in Experiment 3. Note that the luminance profile incorporates the effects of both illumination and reflectance components.

\section{Method}

The same four experimental factors and levels used in Experiments 1 and 2 were repeated in Experiment 3: shape, curvature, illumination, and reflectance of standard patch. The test and standard surrounds were uniform and had the same reflectances as in Experiment 1.

The only difference in Experiment 3 was that the ellipsoids and rectangles were not of uniform albedo but were painted with a series of stripes. Both shapes were horizontally divided into 17 regions that were assigned a random reflectance in the range 0.0-1.0. The result was that the test and standard regions were separated by seven gray stripes. The reflectances of the stripes were random but were the same for corresponding conditions involving ellipsoids and rectangles. For example, for the high-curvature, side-illumination, and high-standard patch condition, both ellipsoids and rectangles were displayed with the exact same set of gray stripes.

\section{Results and Discussion}

The results of Experiment 3 are shown in Figure 9. Overall performance was worse than that in Experiment 1.

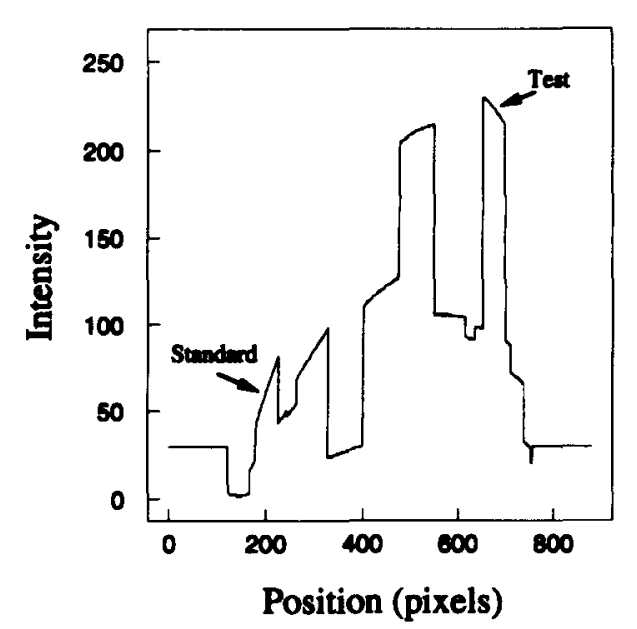

Figure 8. Example of luminance profile used in Experiment 3 (in arbitrary units). The object was defined as having side illumination, high curvature, and high reflectance standard (compare with left profile in Figure 4). 
Table 1

Mean Errors for Experiments 1-3

\begin{tabular}{cccc}
\hline & \multicolumn{3}{c}{ Subject } \\
\cline { 2 - 4 } Experiment & A.G. & N.M. & L.P. \\
\hline 1 & 0.123 & & 0.150 \\
2 & 0.089 & 0.109 & 0.097 \\
3 & 0.190 & 0.217 & \\
\hline
\end{tabular}

Note-For each subject and experiment, entries were obtained by summing up an error index for all experimental conditions. Entries were normalized by dividing the sum by the total number of trials (conditions $X$ repetitions). For a fixed condition, the error index was given by the absolute value of the difference of the log of the observer setting and the log of the associated standard patch reflectance.

For example, Subject N.M. had mean matches as low as $R=0.65$ for the $R=0.95$ standard and $R=0.36$ for the $R=0.5$ standard; Subject A.G. had mean matches of $R=$ 0.22 for the $R=0.30$ standard. The poorer constancy obtained in Experiment 3 is best illustrated by considering the matches for ellipsoids of low and high curvature (first and second pair of points). For Subject N.M., lightness matches for high-curvature ellipsoids were lower than for low-curvature ones for all standard patch reflectances. For Subject A.G., this same trend can be observed for the $R=0.95$ standard patch, as well as other specific matches (for $R=0.5$, compare the second setting with the fourth; for $R=0.3$, compare the first setting with the third). This behavior should be contrasted to the respective matches for Experiment 1, especially for Subject A.G. (Figure 5). It should be noted that this deviation from constancy is in the direction of luminance matches; high-curvature objects produce much more extreme variation in the luminances of standard and test patches of same reflectance (see Figure 4).

It is useful to explicitly compare the performance obtained in Experiments 1-3. Table 1 shows an error measure computed for each experiment and subject based on the differences between a subject's match and the associated standard patch reflectance for all experimental conditions. Constancy was best in Experiment 2, followed by Experiment 1, and was worst in Experiment 3. Especially interesting is the case of Subject A.G., who ran all three experiments.

Although Experiment 3 errors correspond to mismatches only on the order of 1.0-1.5 Munsell steps, it should be remembered that the high-standard $(R=0.95)$ and medium-standard $(R=0.50)$ conditions were increments, with the test surrounds fixed at $R=0.4$ and $R=$ 0.1 , respectively, which limits the range of possible matches (e.g., increments will tend to be matched to increments) ${ }^{8}$ Again, object shape did not have a large effect on lightness matches but was statistically significant [for Subject A.G., $F(1,72)=4.41, p<.04$; for Subject N.M., $F(1,72)=11.06, p<.0015$ ]; compare lightness matches for Subject N.M. for the $R=0.95$ and $R=$ 0.30 standards (compare the first pair of settings to the third, and the second to the fourth). Curvature was significant [for Subject A.G., $F(1,72)=5.31, p<.03$; for Subject N.M., $F(1,72)=27.92, p<.0001]$. As before, the illumination direction was not statistically significant. It should be pointed out that the subjects' reports indicate that some of the lightness matches of Experiment 3 were harder to perform than those in the previous experiments. This is reflected in the larger error bars seen in Figure 9 (see the Individual Differences section below for further discussion).

In Experiment 3, we expected a more substantial difference in the lightness settings between the ellipsoidal and flat displays, given that the objects used contained reflectance stripes. For the flat displays, the luminance gradients due to the reflectance changes were exactly parallel to those from the shading, whereas, for the ellipsoids, the vertical component of the shading provided illumination information that was not confounded with reflectance gradients. However, as mentioned above, object shape did not have a large effect on lightness matches.

In a study of the 3-D shape induced on flat displays by space-modulated illumination patterns projected onto them, Bergström, Gustafsson, and Jakobsson (1993) found that structure (number of reflectance/illuminance edge intersections) was an important factor facilitating the distinctness of the induced 3-D shape (see also Bergström, Gustafsson, \& Putaansuu, 1984, and discussion below). Assuming that, in our objects, better 3-D perception is coupled with better lightness constancy, Bergström et al.'s results may, on the surface, indicate that the con-

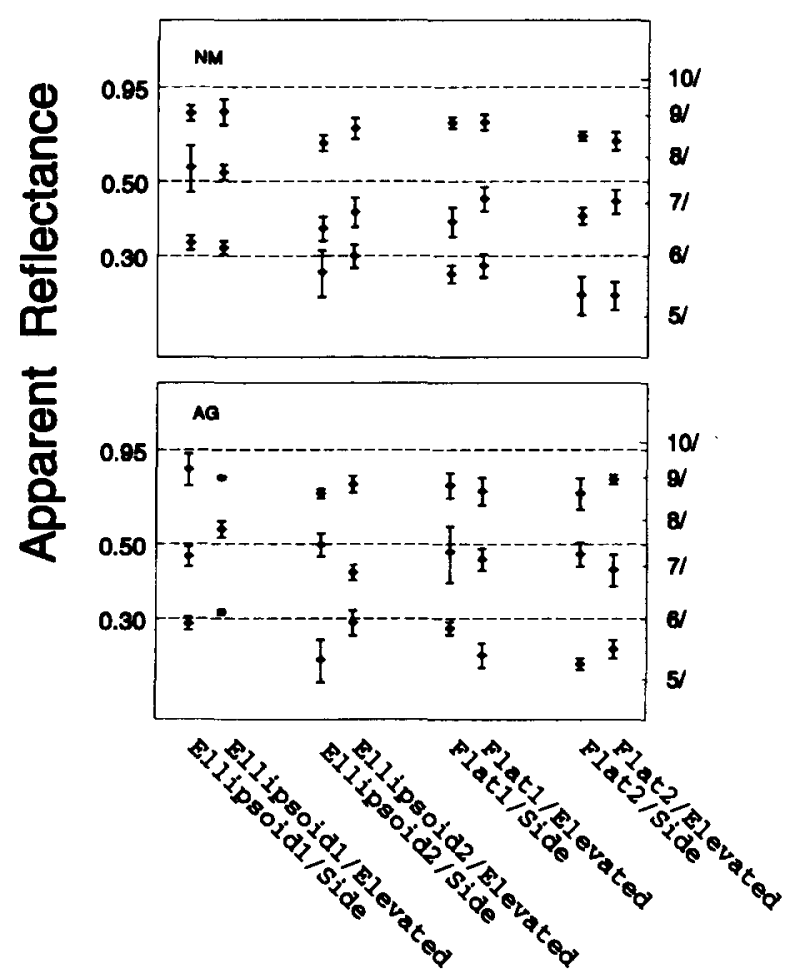

Figure 9. Experiment 3. Lightness matches in log coordinates for Subjects N.M. and A.G. Munsell values are given on the right for reference. Conditions as in Figure 5. Matches are the mean of several presentations; $n=4$ for Subject N.M., and $n=4$ for Subject A.G. Error bars denote \pm 1 standard error. 
ditions of Experiment 3 would optimize lightness constancy, since the vertical arrangement of the stripes produces a more structured display. However, as these authors state, the "spatial modulation of illumination always informs about the 3-D layout, provided that the modulation is not mistaken for a reflectance gradient" (Bergström et al., 1993, p. 656, italics added). In our curved-in-depth displays, a vertical arrangement of stripes accentuates the conditions for the illumination and reflectance components over the objects to be entangled. In fact, the experiment was designed to produce a stricter test of the capabilities of the visual system to disentangle these two contributions to image formation.

In summary, the poor performance in Experiment 3 indicates that the parsing of luminance gradients into illumination and reflectance components was not properly attained in the objects used. Further experiments will have to investigate whether performance is better in scenes with richer 3-D structure (e.g., objects with hills and valleys).

\section{GENERAL DISCUSSION}

We studied the perception of lightness in 3-D objects, extending the investigation of constancy to scenes where illumination varies due to changes in shape. Lightness constancy requires that the visual system appropriately parse the input scene into illumination and reflectance components. The results of Experiments 1 and 2 showed that the visual system was able to perform such operation for our ellipsoid shapes. In other cases, significant deviations occurred (e.g., some matches for rectangles in Experiments 1 and 3).

One question explored in Experiments 1-3 was whether the perception of 3-D surface and illumination layout help calibrate lightness judgments. Our results showed a small but consistent improvement between lightness matches on ellipsoid shapes, relative to flat rectangle shapes under similar illumination conditions. The shading employed on rectangles was unavoidably equivalent to that of a cylinder. As such, the illumination provided cues for a 3-D cylindrical object, although the surface geometry did not correspond to a cylinder. It is possible, then, that the small effect of shape revealed in the present experiments was due to the 3-D shading cues present on our "flat" objects. In other words, rectangle objects also contained powerful cues as to illumination change over a "3-D form."

It should be pointed out that ellipsoids and flat rectangles differed in two important respects: (1) in having different bounding contours, and (2) in the number of directions along which luminance varied over the object's surface--horizontal and vertical for ellipsoids and horizontal only for rectangles. Thus, the observed improvement in lightness constancy for the 3-D ellipsoids over the rectangles may be related to the number of directions in which the luminance varies in the image or the shape of the bounding contour, or both. The present experiments do not distinguish between these two possibilities. Our main concern, at present, is not to make claims about which factors for conveying information about 3-D surface curvature and illumination intensity can impact lightness constancy but rather to demonstrate that it is possible to improve performance in lightness constancy when comparable image gradients are embedded in images depicting curved 3-D objects, as opposed to flat (planar) surfaces.

\section{Lightness Constancy: 3-D Spatial Arrangement and Depth}

The connection between color (or lightness) constancy and depth perception was demonstrated by Mach (1886/ 1959) using a folded card with one half illuminated and the other shaded. When seen as flat, the card appeared to have different colors on the two halves, whereas, when seen as folded, the two halves looked the same color but differently lit (Figure 10; see the first figure in Gilchrist, 1979 , for a similar example using a natural scene). Since Mach's demonstration, many researchers have probed the dependency between lightness perception and depth perception; some of the first investigations studied versions of the Mach card illusion itself (Beck, 1965; Hochberg \& Beck, 1954; Katona, 1935; see also Beck, 1972). Our own work reported here can be viewed as continuing this line of investigation. Here, we briefly review a few related studies.

Bergström (1982, 1994; Bergström et al., 1984) performed a series of experiments where square-wave gratings were projected onto two different types of surface: a simplified chromatic Mondrian and a homogeneous

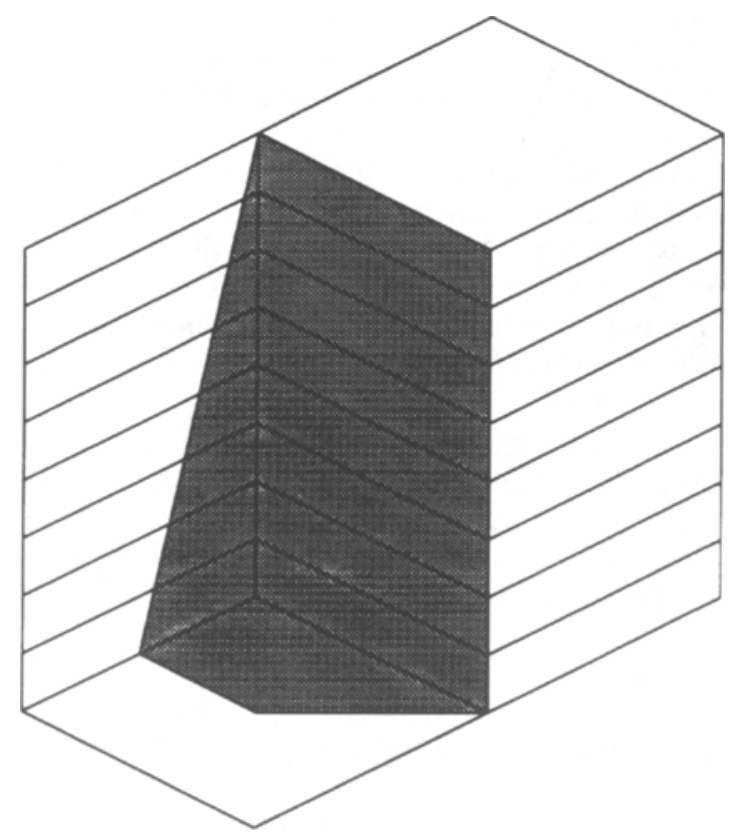

Figure 10. Mach card illusion (Marr's, 1982, version). The perceptual interpretation of the brightness gradient changes when depth reverses. In one depth relation, the gradient is of apparent illumination; the dim area is seen as the shadow of a vertical white face - thus, lightness constancy occurs. In the other, it is a gradient of lightness; one sees a wedge-shaped object with gray surfaces. 
gray surface. Observers were asked to indicate whether they perceived the test surface (Mondrian or homogeneous surface with superimposed illumination grating) as a 2-D (striped) surface or as a 3-D (pleated) object. Bergström et al. (1984) found frequencies of 3-D responses significantly higher for the Mondrian surface than for the homogeneous gray surface. The results were interpreted as indicating better color constancy in the articulated Mondrian condition since the dark phases of the grating were most likely interpreted as attached shadows.

Gilchrist $(1977,1980)$ investigated the dependence of perceived lightness on perceived spatial arrangement. He was able to show dramatic shifts in lightness (from black to white) of the same target by having it observed under monocular and binocular viewing. The different spatial layouts perceived in these two conditions were thus coupled with rather distinct lightness assignments. In yet another striking demonstration, Gilchrist showed the effect of depth on lightness perception (Gilchrist, 1977). In this study, he employed a peephole arrangement in which certain parts were illuminated by carefully hidden light sources. The target, consisting of a piece of white cardboard, could appear in a near plane or in a far plane depending on the manipulation of interposition cues. With this arrangement, the same white target appeared almost black or white depending on where (near or far plane) it was perceived. Gilchrist's study of depth showed that luminance ratios within perceived depth planes, and not retinal ratios, are important in determining lightness, the "coplanar ratio hypothesis" (see also Wist, 1974; Wist \& Susen, 1973). Gilchrist's depth experiments were later replicated on a CRT by Schirillo et al. (1990), who showed that lightness, but not brightness, depends on perceived depth information (see also Schirillo \& Arend, 1995; Schirillo \& Shevell, 1993).

Recent studies have also shown that the perception of 3-D curved shapes interacts with lightness perception. Knill and Kersten (1991) showed that the lightness of a stimulus can be determined by the shape of its outline contour (see also Ramachandran, 1988, and Pessoa et al., 1995). More specifically, they found that the CraikO'Brien-Cornsweet effect can be eliminated when the contours are curved. Such stimulus is perceived as two 3-D abutting cylinders, and, according to Knill and Kersten, the luminance variation due to the "cusp" is interpreted as being caused by shading across a 3-D surface of uniform reflectance, or lightness. Buckley et al. (1994) have replicated Knill and Kersten's results and shown that similar effects can be elicited by "shape from stereopsis."

\section{Discounting the Illuminant}

Many theories of lightness constancy assume an initial stage of "discounting the illuminant," which discards information about absolute luminance and renders lightness dependent on local luminance ratios (e.g., Cornsweet, 1970; Grossberg \& Todorović, 1988; Land \& McCann, 1971; Shapley \& Enroth-Cugell, 1984). The mechanisms proposed to accomplish this task are basically instantiations of Wallach's (1948) ratio hypothesis. However, as has been shown experimentally before, the visual system does not "just" discard the illuminant information. For example, in Arend and Goldstein's (1990) illumination gradient study, lightness matches were illumination invariant, but brightness matches varied substantially with illumination. Furthermore, although in this task observers had excellent constancy, the displays contained clearly visible illumination gradients. As Gilchrist (1994, p. 20) has pointed out, lightness constancy matches are not metameric matches.

It is interesting that the illumination information that is discarded by most theories of lightness constancy is absolutely essential to "shape-from-shading" algorithms (see Horn \& Brooks, 1989) that recover shape from the illumination information. In fact, such algorithms require that gradients of surface reflectance do not contribute to the luminance gradients in the input image. Either objects must be of uniform reflectance or the reflectance gradients must be known in advance (so that they can be taken into account).

The ellipsoid objects studied in the present paper pose a challenge to both theoretical traditions mentioned above. The illumination gradients were clearly visible, causing large variations of brightness over the solids. As stated, brightness matches were not collected. However, when asked to compare the brightnesses of the test and standard regions when their appearances were set by the subjects' previous lightness matches, the observers reported large deviations (see also the discussion of Experiment 1). Given the results of Arend and Goldstein (1990) on unevenly illuminated scenes, brightness matches on the present objects should also vary with luminance. Moreover, in Experiment 3, the illuminated objects were not of uniform albedo but contained a series of gray stripes, violating one of the main assumptions of shape-from-shading algorithms.

In Land and McCann's (1971) classic demonstration of lightness constancy, a Mondrian display was illuminated in such a way that a gray patch at one extreme of the display reflected the same mean luminance as a white field on the other extreme. According to Land and McCann, that the gray field looked gray and the white field looked white was the result of the low sensitivity of the visual system to shallow luminance gradients. ${ }^{10}$ Bergström $(1977,1982,1994)$ has argued, however, that perceiving the illumination is a condition for proper surface color perception, not the other way around. In his vector model, it is the fact that the gradual variation of luminance is recognized (i.e., perceived) as a common component (i.e., as illumination) that allows lightness constancy. The present results supply evidence that some information concerning illumination change over 3-D forms not only is not discarded but is taken into account, given that performance with ellipsoid and rectangle objects differed.

\section{Lightness Theories and Multidimensional Representation}

As stated, our ellipsoids contained both visible illumination and lightness components. As such, they provide excellent examples of the multidimensional nature 
of surface color perception (Arend, 1994; Gilchrist, 1994). Current lightness theories are incomplete since they provide no mechanism for representing these two dimensions of surface perception - not to mention more complicated phenomena that are also common, such as transparency, visible light sources, highlights, and reflections (for some ideas of how this may be accomplished, see Arend, 1994).

Even more vexing is that current theories provide no means for distinguishing illumination gradients from reflectance gradients. Gradient-integration algorithms propose the use of a threshold in order to eliminate shallow spatial gradients, and some authors (Horn, 1974; Land \& McCann, 1971) have suggested that this threshold can be used to separate illumination and reflectance components. While such strategy may suffice in some situations, it is clearly not sufficient in general.

\section{Illumination Gradients}

Arend and Goldstein (1990) investigated lightness and brightness perception in scenes with illumination gradients. Excellent lightness constancy was reported for all linear and slant luminance profiles in both Mondrian and uniform displays. Some of the conditions of Experiment 1 did not produce such degree of constancy and may seem at odds with the Arend and Goldstein (1990) report. Note, however, that, in the experiments of Arend and Goldstein (1990), the standard reflectance was fixed at 0.5 . When the standard was $R=0.5$ in the present Experiment 1 (Figure 5), lightness matches followed those expected for constancy. The largest errors were settings of $R=0.42$, corresponding to deviations less than half of a Munsell step. Moreover, note that, in Experiment 1, different standard and test surround reflectances prevented local apparent contrast matches from giving lightness constancy matches. It is possible that, in the study of Arend and Goldstein (1990), subjects used local luminance ratios as indications for lightness matches and therefore obtained excellent results. The present study shows, however, that good constancy may be obtained when subjects cannot resort to this strategy, as the performance with the $R=0.5$ standard attests. For the other standard reflectances, some larger deviations occurred.

\section{Relative Versus Absolute Lightness}

An observer has relative lightness constancy if he/she has interval-scale knowledge of reflectance (i.e., if he/she can tell that a patch under one illuminant has a higher reflectance than a second patch under a different illuminant and by how much). If, in addition, he/she assigns the name "black" to low physical reflectance $(2 \%-5 \%)$, "white" to high physical reflectance $(80 \%-95 \%)$, and appropriate gray estimates for intermediate reflectances, then he/she has absolute lightness constancy.

Before discussing whether the present results reveal relative or absolute lightness constancy, it is important to question what kind of judgment the subjects performed. In particular, were they assessing apparent surface color (i.e., lightness)? It should be noted that many of the ex- periments widely interpreted as studies of lightness constancy have failed to provide the information necessary to establish that the subjects were manipulating apparent surface colors (e.g., Cornsweet, 1970; Jameson \& Hurvich, 1961). The best way to ensure that subjects are judging apparent color is to have them perform both brightness and lightness matches (see Arend \& Golstein, 1987; Jacobsen \& Gilchrist, 1988). Although our subjects were not required to do both kinds of matches, there are several lines of evidence that indicate that they were assessing surface properties. First, given that in our displays the simulated illumination gradients looked more like illumination gradients than reflectance gradients, and vice versa, we can assume that our subjects were able to follow the instructions to make the patches appear to have been cut from the same paper. Second, and most compelling, evidence for the evaluation of surface properties comes from the realization that apparent contrast (local ratio) matches do not produce our results (see our discussion of Experiment 1).

Insofar as our matches reflect judgments of surface properties, we can conclude that, in the conditions where good constancy was obtained (e.g., Experiment 2), the results reflect the accurate matching of relative reflectance (i.e., of relative lightness constancy). However, they do not demonstrate absolute lightness constancy. To establish this, it is necessary to adopt a procedure in which the subject reports not just the stimulus settings that have the same shade of gray but also which shade of gray is perceived. In fact, there is some evidence that, in some cases, absolute lightness constancy did not occur. For example, when asked about the color of the $R=0.95$ standard in Experiment 1, more often than not, colleagues around the lab would report that they appeared to be a light gray patch-remember that the standard was always positioned away from the light source. ${ }^{11}$ In the study of Arend and Goldstein (1990), the fact that the $R=0.95$ simulated patch looked white (and the $R=0.03$ patch looked black) was taken as evidence that their data indicate absolute lightness constancy. For our studies with curved objects, further tests with a Munsell chart are needed to establish whether or not the lightness matches found reflect absolute lightness constancy.

We are not the first investigators to distinguish between relative and absolute lightness. Evans (1974) made a similar observation and defined relative and absolute lightness accordingly (see also Arend \& Goldstein, 1987, 1990).

\section{Individual Differences}

During the discussion of the results of the three experiments presented here, we have noted some individual differences in the lightness matches produced. For example, in Experiment 1, for the low-reflectance condition, Subject L.P. tended to underestimate the standard reflectance, whereas Subject A.G. went from small overestimations for low-curvature ellipsoids to small underestimations for rectangles with elevated illumination and high curvature. It is possible that, for some trials, indi- 
vidual differences stem from different strategies in performing the task. For example, upon completing Experiment 3, without being prompted, Subject A.G. reported that for some stimuli he found the task rather hard because the direction of illumination was not evident for the stimuli and that, in such cases, he resorted to the "strategy" of assuming the light source to the right of the objects, as verbally indicated to the subjects in the beginning of the session.

The present experiments require the subjects' parsing of the input scene into illumination and reflectance components. In this context, the individual differences found may indicate variations in the way these components are attributed by each individual given her or his assessment of the precise illumination direction, object shape, and the steepness of the illumination gradient on the objects. ${ }^{12}$ Given the binocular natural viewing mode employed by the subjects, it is also possible that different eye-movement patterns are related to this assignment (see Arend \& Spehar, 1993a, p. 454, for a discussion on eye movements). Nevertheless, we stress that the similarities in the overall behavior of the 3 subjects far outweigh the discrepancies revealed. Thus, lightness constancy was quite good when a rich local gray-level context was provided (Experiment 2), whereas larger deviations occurred when both illumination and reflectance changed along the surface of the objects (Experiment 3 ).

\section{CONCLUSION}

In these experiments, we studied the perception of lightness in 3-D curved objects. Lightness constancy requires that the visual system appropriately decompose the input scene into illumination and reflectance components. The result of the present experiments showed that, although the visual system was able, in some cases, to perform such operation in our 3-D objects, in others, deviations occurred. Lightness constancy was quite good when a rich local gray-level context was provided (Experiment 2). Deviations occurred when both illumination and reflectance changed along the surface of the objects (Experiment 3 ).

One of the questions addressed in this study was whether the perception of surface and illuminant layout help calibrate lightness judgments. Does the perception of 3-D shape and illumination layout reorganize the parsing of the scene into illumination and reflectance components? The difference in performance between ellipsoid and rectangle objects provided evidence that illumination change in 3-D form is taken into account. However, the effect of shape on lightness matches was weak. Ellipsoids are simple shapes that are not very rich in shading information, and the flat shading is inevitably almost as rich when one controls for the illumination distribution along one dimension of such objects, as presently done. Further studies with more general shapes (e.g., involving bumps and hills) and lighting conditions (e.g., shadows) may reveal a larger role for the perception of surface and illumination layout in calibrating lightness.

\section{REFERENCES}

AREND, L. [E.] (1994). Surface colors, illumination, and surface geometry: Intrinsic-image models of human color perception. In A. L. Gilchrist (Ed.), Lightness, brightness, and transparency (pp. 159-213). Hillsdale, NJ: Erlbaum.

Arend, L. [E.], \& Goldstein, R. (1987). Simultaneous constancy, lightness, and brightness. Journal of the Optical Society of America $A, 4,2281-2285$.

AREND, L. [E.], \& GoLDSTEIN, R. (1990). Lightness and brightness over spatial illumination gradients. Journal of the Optical Society of America $A, 7,1929-1936$.

AREND, L. E., \& SPEHAR, B. (1993a). Lightness, brightness, and brightness contrast: 1. Illuminance variation. Perception \& Psychophysics, 54, 446-456.

AREND, L. E., \& SPEHAR, B. (1993b). Lightness, brightness, and brightness contrast: 2. Reflectance variation. Perception \& Psychophysics, 54, 457-468.

BECK, J. (1965). Apparent spatial position and the perception of lightness. Journal of Experimental Psychology, 69, 170-179.

BECK, J. (1972). Surface color perception. Ithaca, NY: Cornell University Press.

Bergström, S. S. (1977). Common and relative components of reflected light as information about the illumination, colour, and threedimensional form of objects. Scandinavian Journal of Psychology, 18, 180-186.

BERGSTRÖm, S. S. (1982). Illumination, color, and three-dimensional form. In J. Beck (Ed.), Organization and representation in perception (pp. 365-378). Hillsdale, NJ: Erlbaum.

BergSTRÖM, S. S. (1994). Color constancy: Arguments for a vector model for the perception of illumination, color, and depth. In A. L. Gilchrist (Ed.), Lightness, brightness, and transparency (pp. 257-286). Hillsdale, NJ: Erlbaum.

Bergström, S. S., Gustafsson, K.-Ą., \& Jakobsson, T. (1993). Distinctness of perceived three-dimensional form induced by modulated illumination: Effects of certain display and modulation conditions. Perception \& Psychophysics, 53, 648-657.

Bergström, S. S., Gustafsson, K.-A., \& Putaansul, J. (1984). Information about three-dimensional shape and direction of illumination in a square-wave grating. Perception, 13, 129-140.

BuCKLEY, D., Frisby, J. P., \& FreEMAN, J. (1994). Lightness perception can be affected by surface curvature from stereopsis. Perception, 23, 869-881.

Cornsweet, T. N. (1970). Visual perception. New York: Academic Press.

Evans, R. M. (1974). The perception of color. New York: Wiley.

Gilchrist, A. L. (1977, January 14). Perceived lightness depends on perceived spatial arrangement. Science, 195, 185-187.

GiLCHRIST, A. L. (1979). The perception of surface blacks and whites. Scientific American, 240, 112-123.

Gilchrist, A. L. (1980). When does perceived lightness depend on perceived spatial arrangement? Perception \& Psychophysics, 28, 527 538.

GILCHRIST, A. L. (1988). Lightness contrast and failures of constancy: A common explanation. Perception \& Psychophysics, 43, 415-424.

GiLCHRIST, A. L. (1994). Introduction: Absolute versus relative theories of lightness perception. In A. L. Gilchrist (Ed.), Lightness, brightness, and transparency (pp. 1-34). Hillsdale, NJ; Erlbaum.

Gilchrist, A. L., \& CataliotTr, J. (1995). Anchoring of surface lightness values in local and global frameworks. Manuscript in preparation.

Gilchrist, A. [L.], Delman, S., \& Jacobsen, A. (1983). The classification and integration of edges as critical to the perception of reflectance and illumination. Perception \& Psychophysics, 33, 425-436.

Gilchrist, A. [L.], Kossyfidis, C., Bonato, F., Agostini, T., Catal. otTl, J., Li, X., Spehar, B., \& Szura, J. (1995). A new theory of lightness perception. Manuscript in preparation. 
GrossberG, S., \& Todorović, D. (1988). Neural dynamics of 1-D and 2-D brightness perception: A unified model of classical and recent phenomena. Perception \& Psychophysics, 43, 241-277.

HoCHBERG, J. E., \& BECK, J. (1954). Apparent spatial arrangement and perceived brightness. Journal of Experimental Psychology, 47, 263266.

HoRN, B. K. P. (1974). Determining lightness from an image. Computer Graphics \& Image Processing, 3, 277-299.

HorN, B. K. P., \& BrooKs, M. (1989). Shape from shading. Cambridge, MA: MIT Press

JACOBSEN, A., \& Gilchrist, A. (1988). The ratio principle holds over a million-to-one range of illumination. Perception \& Psychophysics, 43, 1-6.

JAMESON, D., \& Hurvich, L. M. (1961). Complexities of perceived brightness. Science, 133, 174-179.

KATONA, G. (1935). Color contrast and color constancy. Journal of Experimental Psychology, 18, 49-63.

KATZ, D. (1935). The world of colour. London: Kegan Paul, Trench, Trubner.

KERSTEN, D. (1991). Transparency and the cooperative computation of scene attributes. In M. Landy \& J. Movshon (Eds.), Computational models of visual processing (pp. 209-228). Cambridge, MA: MIT Press.

KNILL, D. C., \& Kersten, D. (1991). Apparent surface curvature affects lightness perception. Nature, 351, 228-230.

Koenderink, J. J., van Doorn, A. J., \& Kappers, A. M. L. (1994). On so-called paradoxical monocular stereoscopy. Perception, 23, 583594

LAND, E. H., \& McCanN, J. J. (1971). Lightness and retinex theory. Journal of the Optical Society of America, 61, 1-11.

MACH, E. (1959). The analysis of sensations (S. Waterlow, Trans.). New York: Dover. (Original work published 1886)

MARR, D. (1982). Vision. San Francisco: Freeman.

NaKayama, K., \& Shimojo, S. (1990). Toward a neural understanding of visual surface representation. Cold Spring Harbor Symposia on Quantitative Biology, LV, 911-924.

Pessoa, L., Grunewald, A., \& Ross, W. (1995). The Craik-O'Brien lightness effect on 3D surfaces: Curvature and highlights. Investigative Ophthalmology \& Visual Science, 36, S476.

Pessoa, L., \& THOMPson, E. (1995). Finding out about filling-in: A guide to perceptual completion for visual science and the philosophy of perception. Manuscript submitted for publication.

Ramach andRan, V. S. (1988). Perceiving shape from shading. Scientific American, 259, 58-65.

SCHIRILlo, J. A., \& AREND, L. E. (1995). Illumination change at a depth edge can reduce lightness constancy. Perception \& Psychophysics, 57, 225-230

Schirillo, J. [A.], Reeves, A., \& Arend, L. [E.] (1990). Perceived lightness, but not brightness, of achromatic surfaces depends on perceived depth information. Perception \& Psychophysics, 48, 82-90.

SCHIRILlo, J. A., \& ShEvell, S. K. (1993). Lightness and brightness judgments of coplanar retinally noncontiguous surfaces. Journal of the Optical Society of America A, 10, 2442-2452.

Shapley, R., \& EnRoth-Cugell, C. (1984). Visual adaptation and retinal gain controls. In N. Osborne \& G. Chader (Eds.), Progress in retinal research (Vol. 3, pp. 263-346). Oxford: Pergamon.

WALLACH, H. (1948). Brightness constancy and the nature of achromatic colors. Journal of Experimental Psychology, 38, 310-324.

WhitTle, P. (1992). Brightness, discriminability and the "Crispening effect." Vision Research, 32, 1493-1507.

WhitTle, P. (1994). Contrast brightness and ordinary seeing. In A. L. Gilchrist (Ed.), Lightness, brightness, and transparency (pp. 111-157). Hillsdale, NJ: Erlbaum.

WIST, E. R. (1974). Mach bands and depth adjacency. Bulletin of the Psychonomic Society, 3, 97-99.

WIST, E. R., \& SUSEN, P. (1973). Evidence for the role of post-retinal processes in simultaneous contrast. Psychologische Forschung, 36, 112.

\section{NOTES}

1. In this work, we have followed the methodology of Arend and colleagues, who have studied lightness constancy employing binocular presentation in order to preserve spatial relational information available to observers in natural viewing (see Arend \& Goldstein, 1987). Since the present series of experiments reveals for our stimuli a small, but consistent, effect of shape on constancy, it is possible that monocular presentation would have affected the results, inasmuch as they can potentially affect shape perception. However, given that strong shape cues were present in the luminance gradients across our objects, it is likely that the results with monocular presentation would be similar to the ones obtained (see Koenderink, van Doorn, \& Kappers, 1994).

2 . With respect to the screen, the $z$-axis points toward the observer, the $x$-axis corresponds to the horizontal dimension, and the $y$-axis corresponds to the vertical dimension.

3. We employ the words low, medium, and high as a mnemonic of the relative ordering of the reflectances of the standard patches. Note, however, that the lowest reflectance corresponded to that of a Munsell value of 6.0 (middle gray).

4. This was done to avoid ceiling effects in the bracketing procedure of the adjustments. Reflectances moderately greater than 1.0 did not appear luminous in our displays, and the observers never chose a final setting greater than $R=1.06$.

5. The results reported in the paper are from multiway ANOVA tests for individual subjects.

6. Given that test and standard patches exhibited luminance gradients, reflectance settings corresponding to luminance predictions are based on the center position of the test and standard patches.

7. Here and in other places, reflectances were randomly drawn from the set $[0.0,1.0]$. As discussed in the General Method section, the lighting model was Lambertian, with a small ( $5 \%$ ) component of ambient illumination. The ambient illumination prevented image regions of low reflectance from having luminance levels too close to zero.

8. In the present series of experiments, increments were never matched to decrements. We believe that this behavior stems from the choice of reflectances employed. For example, for the medium-reflectance standard of $R=0.5$, the test surround was $R=0.1$ and the standard surround was $R=0.2$. Matching the increment of the patch-surround standard configuration to a decrement of the test configuration would require that the setting be less than $R=0.1$, which is a rather unlikely match. However, pilot studies have revealed that increment-decrement matches are possible using our ellipsoidal stimuli.

9. It should be noted that several casual observers reported, on first impression, the perception of curved surfaces for rectangle objects (see Figure 3, middle; the reader should be reminded of the impoverishment due to photographic reproduction).

10 . It is unlikely that the visual system's sensitivity in perceiving luminance gradients plays an important role in lightness constancy. Excellent lightness constancy was obtained by Arend and Goldstein (1990) in the presence of abrupt, visible illumination gradients.

11. Pilot studies have revealed that interchanging the positions of the test and standard patches does not alter the general pattern of results.

12. We remind the reader that only Subject L.P. was experienced in lightness matching. While the question of whether the subjects' matches reflect more "judgment" than "perception" cannot be easily answered, it is reassuring that subjects with rather distinct levels of experience produced very similar results. An interesting point is that experienced Subject L.P. was the one who produced the largest deviations from constancy in Experiment 2; Subject L.P. commonly underestimated the low-reflectance standard. These considerations suggest that our matches reflect "perception" more than "judgment." In any case, the issue is worth considering and has prompted some researchers to probe lightness constancy by other means (e.g., Kersten, 1991). Pessoa and Thompson (1995) address some of these issues in the context of perceptual "filling-in."

(Manuscript received August 7, 1995; revision accepted for publication February 5, 1996.) 